\title{
Raft association and lipid droplet targeting of flotillins are independent of caveolin
}

\author{
Rajendran, L ; Le Lay, S ; Illges, H
}

DOI: https://doi.org/10.1515/BC.2007.034

Posted at the Zurich Open Repository and Archive, University of Zurich ZORA URL: https://doi.org/10.5167/uzh-49612

Journal Article

Published Version

Originally published at:

Rajendran, L; Le Lay, S; Illges, H (2007). Raft association and lipid droplet targeting of flotillins are independent of caveolin. Biological Chemistry, 388(3):307-314.

DOI: https://doi.org/10.1515/BC.2007.034 


\section{Raft association and lipid droplet targeting of flotillins are independent of caveolin}

\author{
Lawrence Rajendran ${ }^{1,2, \star}$, Soazig Le Lay ${ }^{1}$ and \\ Harald Illges ${ }^{2}$ \\ ${ }^{1}$ Max Planck Institute of Molecular Cell Biology and \\ Genetics, Pfotenhauerstr. 108, D-01307, Dresden, \\ Germany \\ ${ }^{2}$ Immunology, Department of Biology, University of \\ Konstanz, D-78457, Konstanz, Germany \\ ${ }^{*}$ Corresponding author \\ e-mail: rajendra@mpi-cbg.de
}

\begin{abstract}
Lipid rafts are liquid ordered platforms that dynamically compartmentalize membranes. Caveolins and flotillins constitute a group of proteins that are enriched in these domains. Caveolin-1 has been shown to be an essential component of caveolae. Flotillins were also discovered as an integral component of caveolae and have since been suggested to interact with caveolins. However, flotillins are also expressed in non-caveolae-containing cells such as lymphocytes and neuronal cells. Hence, a discrepancy exists in the literature regarding the caveolin dependence of flotillin expression and their subcellular localization. To address this controversy, we used mouse embryonic fibroblasts (MEFs) from caveolin-1 knockout $\left(\mathrm{Cav}-1^{-/}\right)$and wild-type mice to study flotillin expression and localization. Here we show that both membrane association and lipid raft partitioning of flotillins are not perturbed in Cav-1/- MEFs, whereas membrane targeting and raft partitioning of caveolin-2, another caveolin family protein, is severely impaired. Moreover, we demonstrate that flotillin-1, but not flotillin-2, associates with lipid droplets upon oleic acid treatment and that this association is completely independent of caveolin. Taken together, our results show that flotillins are localized in lipid rafts independent of caveolin-1 and that translocation of flotillin-1 to lipid droplets is a caveolin-independent process.
\end{abstract}

Keywords: caveolae; caveolin; flotillin; lipid bodies; lipid droplets; rafts; reggie.

\section{Introduction}

Lipid-lipid immiscibility in cellular membranes imparts heterogeneity in the membrane plane and a subset of these lateral heterogeneities is termed lipid rafts (Rajendran and Simons, 2005). Lipid rafts are submicroscopic dynamic assemblies, which are more tightly packed than the surrounding liquid disordered regions (Simons and Vaz, 2004). Rafts can be biochemically defined as detergent-resistant membranes (DRMs) and hence can be isolated as DRM fractions using flotation gradients. These domains have been implicated in several key cellular functions such as signaling, endocytosis, polarization (Simons and Toomre, 2000). Raft domains have also been shown to be the entry points for viruses (Campbell et al., 2001) and toxins (Lencer, 2001) and to act as pathogenic platforms for conversion of the cellular prion $\left(\mathrm{PrP}^{\mathrm{c}}\right)$ to the scrapie version (PrPsc) (Hooper, 2005), for $\beta$ cleavage of the amyloid precursor protein (APP) (Ehehalt et al., 2003), and for activation of anthrax toxin (Abrami et al., 2003; Kurzchalia, 2003).

The original raft concept proposed that the outer leaflet of the membrane contains lipid raft domains that are enriched in cholesterol and sphingolipids (Simons and Toomre, 2000), but recent evidence points to the existence of an equivalent domain organization in the inner leaflet as well (Rajendran and Simons, 2005). Several proteins have been shown to be partitioned into raft domains in both leaflets. GPI-anchored proteins are arguably well-established raft proteins that segregate into the exoplasmic leaflet and doubly acylated proteins such as the src-tyrosine kinases can serve as markers for the inner leaflet raft domains (Simons and Toomre, 2000). One other well-established inner-leaflet raft protein is caveolin, the scaffolding protein responsible for the formation and stability of caveolae (Parton, 1996). Caveolae are small invaginations on the plasma membrane that are devoid of clathrin and found in many cell types, such as epithelial and endothelial cells, but are clearly absent in neuronal and lymphocytic cells. Caveolin-1, -2 and -3 comprise a group of caveolin family of proteins that act as signature proteins for caveolae (Kurzchalia and Parton, 1999). Caveolin-1 and -3 have been shown to be necessary for the biogenesis of caveolae in vivo (Drab et al., 2001; Galbiati et al., 2001), whereas caveolin-2 is mislocalized in the trans-Golgi network (TGN) in the absence of caveolin-1 and hence is not targeted to the plasma membrane in these cells (Razani et al., 2001; Breuza et al., 2002). Caveolae and caveolins have been implicated in crucial cellular functions such as growth factor signaling, lipid homeostasis and NO signaling (Parton, 2003).

Biochemically, all raft subsets can be purified as one DRM fraction, but single particle tracking and EM studies have shown that different subsets of raft domains exist in the same membrane plane (Edidin, 2003). Caveolae represent a subset of raft domains, but non-caveolae raft domains still exist in both caveolae- and non-caveolaecontaining cells. In mammalian cells, flotillins exist as flotillin-1/reggie-2 and flotillin-2/reggie-1. These proteins are highly homologous and are evolutionarily highly conserved (Malaga-Trillo et al., 2002). Flotillins are palmitoylated and myristoylated proteins that are anchored to the cytoplasmic membrane leaflet (Neumann-Giesen et al., 2004). These proteins were initially discovered as caveolar proteins (Bickel et al., 1997), but at the same time 
were also discovered as reggie proteins that are upregulated during regeneration of goldfish retinal cells (Schulte et al., 1997). The latter study in neuronal cells that lack caveolae and our earlier reports in lymphocytes (Rajendran et al., 2003) also point to the possibility that flotillins are non-caveolar proteins. Therefore, whether flotillins are specifically localized to caveolae and whether caveolins can influence their cellular distribution have not yet been clearly determined.

To determine whether caveolins influence the expression, localization and raft association of flotillins, we used caveolin-1 knockout mouse embryonic fibroblasts (KO MEFs) and studied the localization of flotillins compared to wild-type cells (WT MEFs). We provide biochemical and microscopic evidence that flotillin localization and raft association are independent of caveolin. Moreover, we show that flotillin-1 is recruited to lipid droplets upon fatty acid treatment independently of caveolin-1, whereas flotillin-2 is not. Taken together, our results provide direct evidence that flotillins are non-caveolar proteins with a subcellular organization that is independent of caveolin and could therefore represent a functional subset of noncaveolar rafts.

\section{Results}

\section{Assorted subcellular localization of flotillins in different cell lines}

We recently reported the significance of the asymmetric localization of flotillins in leukocyte signaling. Indeed, most of the flotillins were associated with the plasma membrane and with centrosomes in lymphocytes (Rajendran et al., 2003), as reproduced here in Jurkat cells (Fig- ure 1). To investigate the localization of flotillins in cells of non-lymphoid origin, we studied the expression of flotillins in epithelial-like cells and MEFs. In both cell types, flotillin-1 and -2 were expressed and were of the correct size (Figure 1A). Cellular flotillins were mainly distributed in intracellular structures in HeLa cells and MEFs, but were also strongly evident at the plasma membrane in HeLa cells (Figure 1B). In the case of confluent epithelial cultures, pronounced association of flotillins to the cellcell contact regions could be observed, consistent with earlier reports that flotillins could be involved in cell adhesion processes (Lang et al., 1998).

\section{Caveolin-independent localization of flotillins}

To investigate the requirement for caveolin in flotillin localization, we took advantage of MEFs isolated from cav-1 KO mice, which are fibroblast cells devoid of caveolae (Drab et al., 2001), and compared these with WT MEFs. Both flotillin-1 and -2 were expressed in WT MEFs and showed a punctate pattern (Figures 1 and 2). Caveolin-1 was associated with the plasma membrane and the internal structures, possibly the TGN, endosomes and caveosomes (Figure 2). Although both caveolin-1 and flotillins were present at the plasma membrane, there was almost no overlap between these regions, suggesting that flotillins do not localize preferentially to caveolae. In cav1-KO MEFs, we did not observe any changes in flotillin-1 or -2 expression patterns, despite the absence of caveolin-1 expression, as shown by a lack of cav-1 staining (Figure 2). The fact that a good degree of colocalization of flotillin-1 and -2 was exhibited in both WT and KO MEFs (data not shown) implies that caveolin-1 is not essential for the localization or the colocalization (Figure 2).
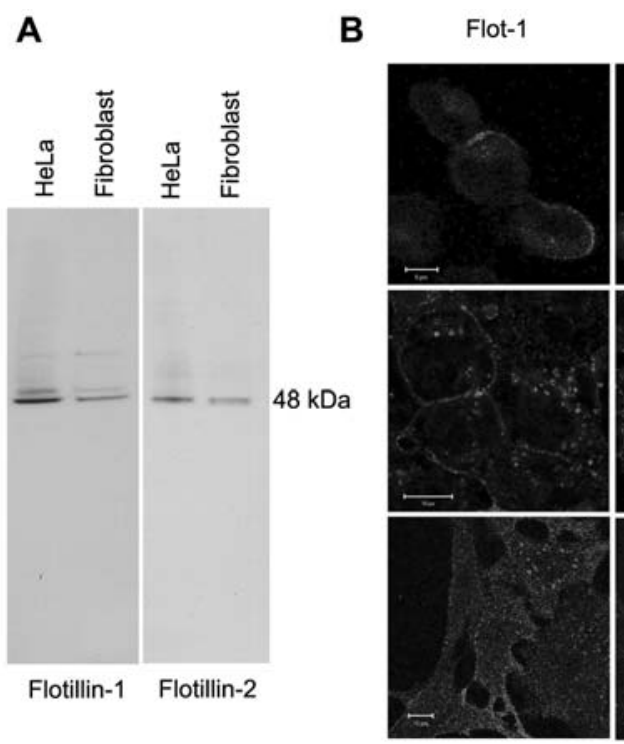

Flot-2

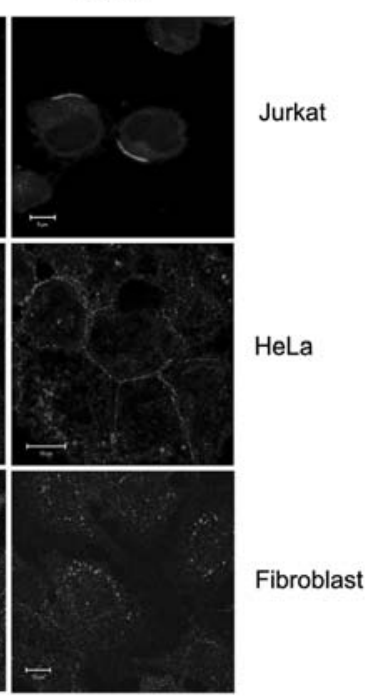

Figure 1 Expression and localization of flotillins.

(A) Western blot analysis of HeLa cells and wild-type MEFs. The left panel shows the blot probed with anti-flotillin-1 antibody and the right blots probed with anti-flotillin-2 antibody and detected with enhanced chemiluminescence detection.

(B) Jurkat, HeLa and wild-type MEFs were immunostained with mouse anti-flotillin-1 (left panel) and mouse anti-flotillin-2 (right panel) and detected with donkey anti-mouse Cy3-conjugated antibodies. Restricted plasma membrane staining is observed for Jurkat cells, whereas HeLa cells presented an assorted pattern both at the plasma membrane and in internal vesicles, and fibroblasts showed mostly intracellular staining. Scale bar represents $5 \mu \mathrm{m}$ for Jurkat and $10 \mu \mathrm{m}$ for HeLa and fibroblasts. 


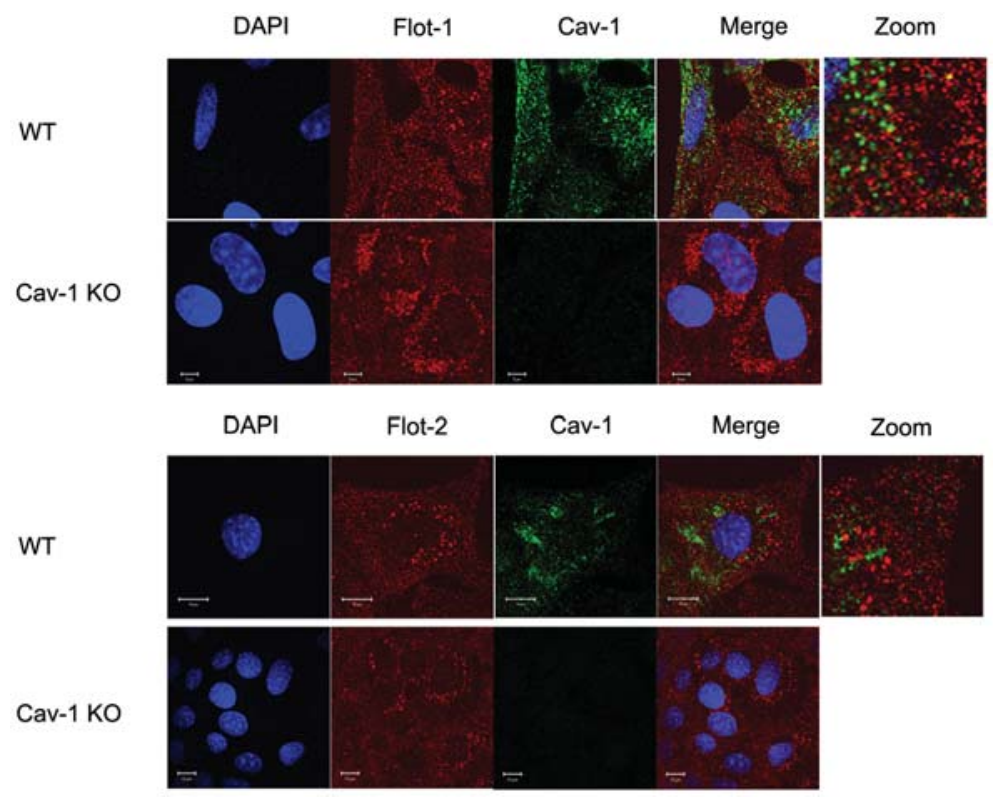

Figure 2 Caveolin-independent flotillin localization.

WT and cav-1 KO MEFs were immunostained for the presence of endogenous caveolin-1 with rabbit anti-caveolin-1 (green) and flotillin-1 with mouse anti-flotillin-1 (upper panel, red; scale bar $5 \mu \mathrm{m}$ ) or flotillin-2 (lower panel; scale bar $10 \mu \mathrm{m}$ ). The nuclei were stained with DAPI. Neither the expression levels nor the localization pattern of flotillins- 1 and -2 changed with respect to the presence of caveolin-1.

\section{DRM association of flotillins is not dependent on caveolin-1 expression}

Since flotillins were previously identified as resident components of caveolae (Bickel et al., 1997) and purification of DRMs containing caveolin resulted in the co-purification of flotillins (Volonte et al., 1999), we tested whether DRMs from cav-1-deficient fibroblasts still retained flotillins. DRMs from WT and KO MEFs were floated on a sucrose gradient after 1\% Triton extraction. WT MEFs DRMs contained almost all of the caveolin-1 and -2 (Figure 3). Flotillin-2 was mostly recovered from DRMs, whereas flotillin-1 was retrieved from both detergent-soluble and -insoluble fractions (Figure 3). On the other hand, most of the caveolin-2 from the cav-1 KO cells

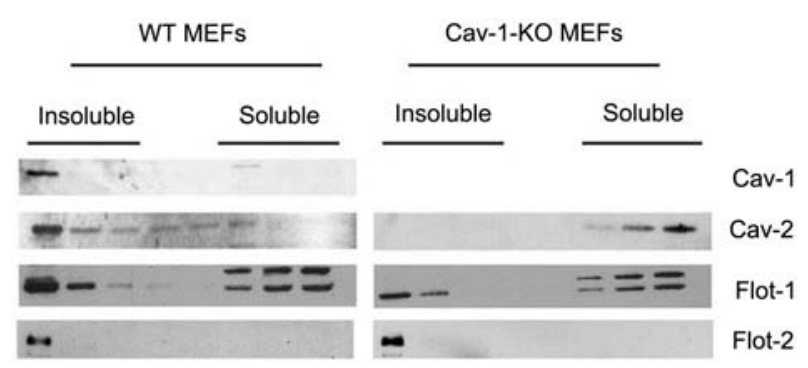

Figure 3 Caveolin-1 is necessary for partitioning of caveolin-2 into DRMs but not for flotillins.

Detergent (1\% Triton X-100)-resistant membranes (DRMs) were isolated from WT MEFs and KO MEFs, blotted and probed for the presence of caveolin-1 (cav-1), caveolin-2 (cav-2), flotillin-1 (flo-1) and flotillin-2 (flo-2). Insoluble fractions refer to the DRMs and the soluble to non-DRM fractions. Only DRM partitioning of Cav-2 was affected by the presence of caveolin-1, whereas flotillin DRM association was not affected by the absence of caveolin-1. remained soluble, consistent with the finding that cav-2 undergoes membrane association in the absence of caveolin-1 and is mainly localized in Golgi in cav-1-lacking cells (Razani et al., 2001). However, there was no notable difference in the DRM residency of both the flotillins in both WT and KO MEFs. This is consistent with our earlier finding that DRM fractions from various leukocytic cells (Rajendran et al., 2003) and neuronal cells (unpublished observations), both of which lack caveolae (Shyng et al., 1994; Fra et al., 1995), contain almost all of the flotillins in DRM fractions.

\section{Lipid droplet association of flotillin-1}

Since caveolins and flotillins are both DRMs markers, we questioned whether these two proteins behave similarly in response to exogenous stimuli. In particular, it was recently described that caveolins can move from the plasma membrane to lipid bodies in response to oleic acid treatment (Pol et al., 2001, 2004). In addition, flotillin-1 has been found in purified lipid droplets formed after fatty acid loading in $\mathrm{CHO}$ cells (Liu et al., 2004). Therefore, we induced lipid droplet formation by oleic acid exposure and studied the association of flotillins with a lipid droplet marker, adipophilin-related protein (ADRP). Treatment of MEFs with oleic acid (OA) resulted in an increase in the size and numbers of lipid bodies, as demonstrated by a drastic increase in ADRP signal. No colocalization between the intracellular structures labeled by flotillins and the lipid bodies either in WT or cav-1 KO MEFs was found (Figure 4A). Interestingly, in these OA loading conditions, flotillin-1 was significantly redistributed to lipid bodies, whereas flotillin-2 remained excluded from these lipid-rich organelles (Figure 4B). In particular, a lack of caveolin-1 did not influence flotillin-1 


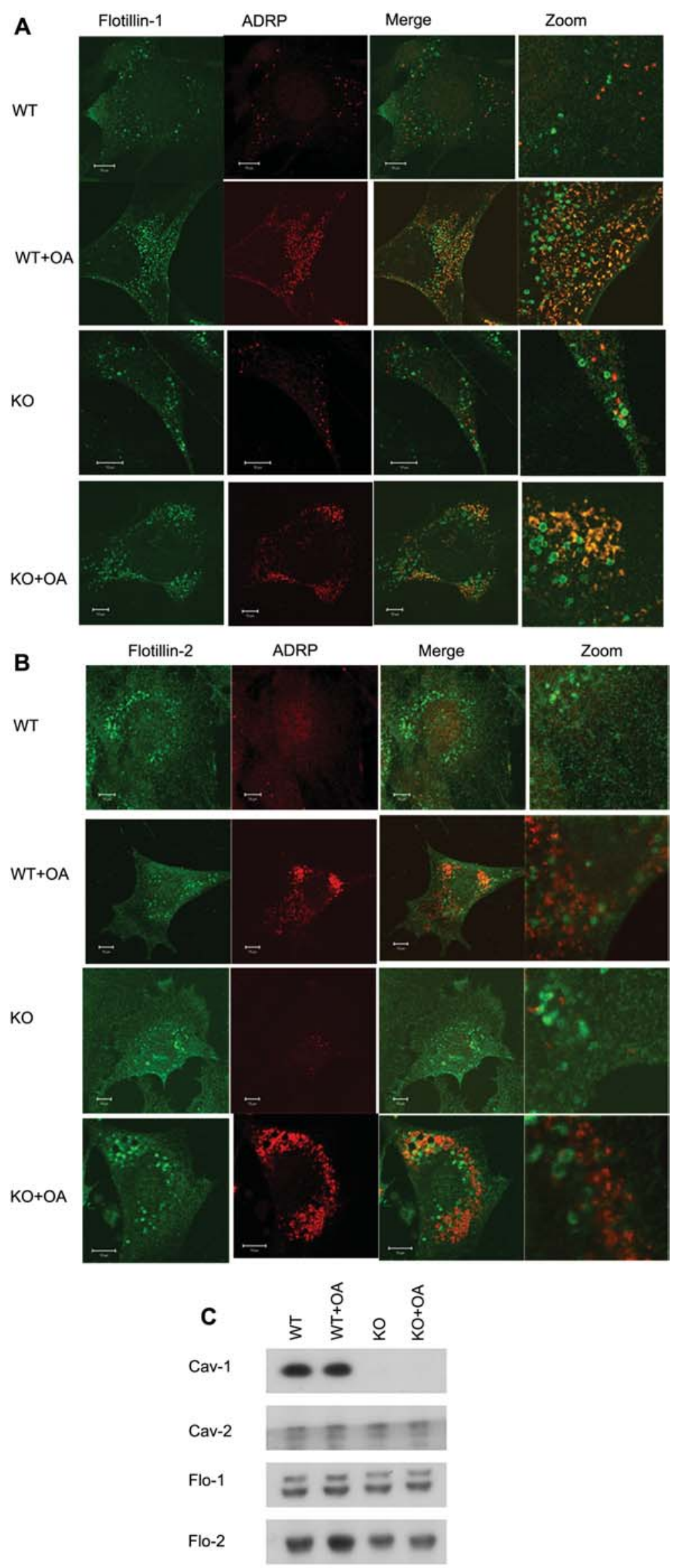


relocalization to lipid bodies. OA treatment of MEFs, however, did not change flotillin or caveolin protein expression drastically, suggesting that this lipid droplet association of flotillin-1 probably results from dynamic targeting from other cell compartments. Therefore, despite their colocalization in unstimulated cells, flotillin-1 and -2 respond differentially to fatty acid stimulation, suggesting functional differences between these two proteins.

\section{Discussion}

Flotillins are raft-associated proteins cloned by three independent groups as proteins involved in neuronal regeneration (Schulte et al., 1997), in epidermal association (Schroeder et al., 1994) and as components of caveolae (Bickel et al., 1997). Flotillins belong to a large family of proteins classified as stomatin/prohibitin/flotillin/ $\mathrm{HfIK/C}$ (SPFH), which is characterized by the presence of a conserved SPFH domain in these proteins (Liu et al., 2005). In mammalian cells, the flotillin protein family includes flotillin-1/reggie-2 and flotillin-2/reggie-1/epidermal surface antigen, which are highly homologous (Malaga-Trillo et al., 2002) and highly conserved across species (Malaga-Trillo et al., 2002). Both flotillins are present in almost all cell types we tested and both are raft-associated, as judged by their association with DRMs (Solomon et al., 2002; Deininger et al., 2003; Rajendran et al., 2003). Flotillins were initially also characterized as co-purifying with caveolae and have been reported to physically interact with caveolin (Bickel et al., 1997). Although these studies showed a caveolar localization of flotillins and their raft association through caveolae, our own work on flotillins in leukocytes (Rajendran et al., 2003) and neurons (Deininger et al., 2003) showed that flotillins are expressed in these cells and raft-associated, despite the fact that these cells lack caveolae. Since the issue of caveolin dependence needed to be addressed in a systematic way, we took advantage of the availability of cav-1 knockout cells.

In the present study, using WT and caveolin-1 KO MEFs, we show that both the expression and localization patterns of the flotillins are independent of caveolins. Although both are localized as punctate structures on the membrane, they are organized as mutually exclusive structures, confirming that flotillins are signature proteins for a subset of non-caveolar proteins. Although detergent treatment disrupts most lipid-lipid interactions, a minor fraction of cell membranes is preserved and could be isolated as DRMs, which are rich in cholesterol and sphingolipids. Caveolins, 'protein' markers of caveolae that represent a subclass of raft domains, are recovered in DRMs. In our study, we demonstrated that the partitioning of flotillins into DRMS or rafts is exclusive of caveolins. It is interesting to note that both caveolins and flotillins in the wild-type MEFs floated to the same buoyant fractions. Earlier studies that reported that flotillins are caveolar-resident proteins and interact with caveolin largely arrived at this conclusion because these two proteins were co-fractionated with caveolins during detergent extraction (Bickel et al., 1997; Volonte et al., 1999). However, DRM association of proteins is only an inclusive criterion for raft localization and does not necessarily separate caveolar from non-caveolar domains (Schuck et al., 2003). Nonetheless, it cannot be ruled out that under certain situations, such as signaling or endocytosis, there could be an interplay between flotillin and caveolins, as suggested by Baumann et al. (2000). These authors reported that upon insulin binding to its receptor, $\mathrm{c}-\mathrm{Cbl}$ and $\mathrm{c}-\mathrm{Cbl}$-associated proteins (CAP) are recruited to the receptor and CAP through its sorbin homology ( $\mathrm{SoHo}$ ) domain binds to flotillin and localizes to caveolae (Baumann et al., 2000). However, under basal conditions, flotillin neither interacts with caveolin nor resides in caveolae. A recent study by Souto et al. (2003) showed that immunopurified rat adipocyte caveolae have a relatively limited protein composition and lack flotillin and insulin receptors. Souto et al. (2003) also failed to coimmunoprecipitate flotillins along with caveolins, reinforcing our findings that flotillins function independent of caveolins.

Another interesting finding from our study is that flotillin-1 is specifically able to redistribute to lipid bodies upon OA treatment, whereas flotillin-2 remains independent from these lipid-rich organelles. Although the two flotillins normally present an overlapping pattern of distribution, this redistribution of flotillin-1 to lipid bodies upon OA stimulation suggests independent and specific functions for each flotillin. Since the mode of membrane anchorage of flotillin-1 (single palmitoylation) is different from flotillin-2, which is myristoylated and multiply palmitoylated, it can be speculated that this could directly influence the capability of these proteins to associate with lipid bodies. Previous proteomic analysis of isolated lipid droplets from $\mathrm{CHO}$ cells already identified flotillin-1 enrichment in these lipid bodies (Liu et al., 2004). Stomatin, a member of the SPFH family, has also been

\footnotetext{
Figure 4 Flotillin-1 but not flotillin-2 is targeted to lipid droplets and the targeting is independent of caveolin-1.

(A) WT and cav-1 KO MEFs were either left untreated or treated overnight with $50 \mu \mathrm{g} / \mathrm{ml}$ oleic acid (OA) to induce lipid droplet formation. The cells were subsequently stained with anti-flotillin-1 (green) and anti-ADRP (red) as a lipid droplet marker. Under basal conditions, small lipid droplets were already detectable, as evidenced by ADRP staining, but flotillin-1 was excluded from these lipidrich organelles in both WT and KO MEFs. OA treatment induced the targeting of flotillin-1 to lipid bodies, as revealed by colocalization of ADRP and flotillin-1 (see merge panel), and was independent of caveolin-1 expression (same pattern in WT or cav-1 KO MEFs). Scale bar, $10 \mu \mathrm{m}$.

(B) Following OA treatment, flotillin-2 remained unassociated with lipid bodies in both WT and KO MEFs; flotillin remained unassociated with lipid droplets. Scale bar, $10 \mu \mathrm{m}$. (C) Whole-cell lysates from basal and OA-treated WT MEFs and cav-1 KO MEFs were electrophoresed, blotted and probed for the presence of caveolin-1, caveolin-2, flotillin-1 and flotillin-2 and visualized by enhanced chemiluminescence. Note that $\mathrm{OA}$ treatment did not influence the expression of any protein.
} 
shown to be targeted to lipid bodies upon expression in MDCK cells (Umlauf et al., 2004). However, this study showed that flotillins were not recruited to lipid bodies, but the authors used a flotillin-2-myc fusion construct to study the targeting of flotillins to lipid droplets, consistent with our finding that flotillin-2 remains unassociated with lipid bodies upon OA treatment. Interestingly, an increasing number of studies have revealed that caveolins can redistribute to lipid droplets under various conditions, including OA treatment (Fujimoto et al., 2001; Ostermeyer et al., 2001; Pol et al., 2001, 2004; Brasaemle et al., 2004). This lipid-body targeting of caveolins is thought to regulate cellular lipid balance, especially cellular cholesterol homeostasis (Martin and Parton, 2005; Pol et al., 2005; Le Lay et al., 2006). We have shown here that flotillin-1 translocation to lipid droplets is independent of caveolin-1.

Currently, the role of flotillin-1 around these lipid-rich organelles is completely unknown. One possibility is that flotillin-1 could induce vesiculation at the plasma membrane upon fatty acid loading. This idea is supported by the fact that flotillin-1 expression in insect cells can induce the formation of large caveolae-like vesicles (Volonte et al., 1999). Moreover, flotillin-1 has recently been described as a determinant of a clathrin-independent endocytic pathway in mammalian cells, which implicates this protein in vesicular trafficking (Glebov et al., 2006). Despite a striking sequence similarity between flotillin-1 and flotillin-2, specific recruitment of flotillin-1 to lipid bodies upon fatty-acid loading could also underline a specific function in lipid regulation or trafficking towards these organelles. Flotillin-1, but not flotillin-2, has been shown to be enriched in maturing phagosomes (Dermine et al., 2001) and exosomes (Rajendran et al., 2006), and only flotillin-1 is specifically upregulated at least 10-fold during adipocyte differentiation (Bickel et al., 1997), suggesting that flotillin-1 could be involved in storage pathways. Finally, another possibility is that flotillins could function as a raft organizer by means of homo/hetero-oligomerization (Neumann-Giesen et al., 2004), and thus would organize or shape scaffold domains at the level of lipid droplet membranes. Indeed, the prohibitin homology $(\mathrm{PHB})$ domain of the flotillin proteins can have a hairpin-like topology, similar to the membrane domain of caveolins. Therefore, it can be speculated that this membrane insertion could influence a positive curvature, as has been described for caveolins, by a local concentration of several hairpin loops inserted in the cytosolic leaflet of the membrane. Our data underline the important functional differences between flotillin-1 and flotillin-2. Flotillin-1, through its unique sequence, may interact with specific proteins and/or lipids and exert its action. Further characterization is needed to elucidate the molecular basis of the functional difference between these two closely related homologs.

\section{Materials and methods}

\section{Cell lines, antibodies and reagents}

The Jurkat T-cell line was from ATCC (Manassas, USA) and was maintained in 5\% FCS containing RPMI at $5 \% \mathrm{CO}_{2}$. Monoclonal antibodies against caveolin-1, caveolin-2, flotillin-1 and flotillin2/ESA were from Transduction Labs/BD Biosciences (Heidelberg, Germany). Polyclonal antibodies against caveolin-1, caveolin-2, flotillin-1 and flotillin-2 were all from Santa Cruz Biotechnology (Heidelberg, Germany). Су3 and Alexa green-conjugated secondary antibodies were from Jackson Laboratories (Dianova, Hamburg, Germany) and Molecular Probes (MoBiTec, Göttingen, Germany), respectively.

\section{Immortalization of MEFs}

Mouse embryonic fibroblasts were prepared from 13.5 p.c. embryos obtained by homozygous crossings of cav- $1 \mathrm{KO}$ mice or WT mice (Drab et al., 2001). MEF cells were immortalized according to the 3 T3 protocol by passaging them continuously until growth rates in culture resumed the rapid rates observed in early-passage MEFs. MEFs were cultured in Dulbecco's modified Eagle medium (DMEM) supplemented with 10\% (v/v) fetal bovine serum, $2 \mathrm{~mm}$ L-glutamine, penicillin and streptomycin (MEF medium).

\section{Isolation of DRMs}

Lipid rafts or DRMs from WT and cav-1 KO MEFs were prepared by discontinuous density gradient ultracentrifugation as previously described (Solomon et al., 2002).

\section{Western blotting}

The gradient fractions, whole-cell lysates of oleic acid-treated or untreated cells or immunoprecipitates were loaded with equal protein amounts on 4-12\% Bis-Tris gradient gels (Novex, Invitrogen, Karlsruhe, Germany) and blotted onto nitrocellulose membranes (Protran, Schleicher \& Schuell, Dassel, Germany) according to the manufacturer's instructions. The blots were blocked twice with $1.5 \%$ BSA/TBST (TBS containing $0.02 \%$ Tween 20) and successively incubated with primary antibodies and HRP-conjugated secondary antibodies (Perbio, Pierce, Heidelberg, Germany). Chemiluminescence was detected using a West Pico Super Signal Chemiluminescence kit (Pierce).

\section{Oleic acid treatment}

WT and cav-1 KO MEFS were incubated for $18 \mathrm{~h}$ in MEF medium containing $50 \mu \mathrm{g} / \mathrm{ml}$ oleic acid (Sigma, Taufkirchen, Germany) conjugated to cyclodextrin.

\section{Immunofluorescence and laser scanning confocal microscopy}

For all fluorescence microscopy experiments, cells were grown on glass coverslips. The cells were then fixed with either icecold methanol for $5 \mathrm{~min}$ at $-20^{\circ} \mathrm{C}$ or $4 \%$ paraformaldehyde for $5 \mathrm{~min}$ at room temperature. After fixation, the cells were washed three times with $0.1 \%$ Triton X-100 containing PBS and then incubated with primary antibodies in $1 \%$ BSA for $1 \mathrm{~h}$ at room temperature. The cells were then washed four times with $0.1 \%$ Triton X-100 containing PBS and the fluorescent-conjugated secondary antibodies were added for $45 \mathrm{~min}$ at room temperature. The cells were subsequently washed three times with PBS and mounted on microscopy slides using Mowiol (Calbiochem, Bad Soden, Germany). After 24 h, the staining was analyzed using an LSM 510 microscope (Zeiss, Oberkochen, Germany) equipped with a Plan-Apochromat $100 \times$ oil immersion lens using the LSM 510 image browser software. 


\section{Acknowledgments}

The authors would like to thank Dr. Temo Kurzchalia for providing cav-1 KO mice and Prof. Kai Simons for advice on the work. We are also grateful to Dr. Juha Torkko and Robin Klemm for critical reading of the manuscript. S.L.L. is a post-doctoral Marie-Curie Fellow (Contract No. MEIF-CT-2003-500768).

\section{References}

Abrami, L., Liu, S., Cosson, P., Leppla, S.H., and van der Goot, F.G. (2003). Anthrax toxin triggers endocytosis of its receptor via a lipid raft-mediated clathrin-dependent process. J. Cell Biol. 160, 321-328.

Baumann, C.A., Ribon, V., Kanzaki, M., Thurmond, D.C., Mora, S., Shigematsu, S., Bickel, P.E., Pessin, J.E., and Saltiel, A.R. (2000). CAP defines a second signalling pathway required for insulin-stimulated glucose transport. Nature 407, 202-207.

Bickel, P.E., Scherer, P.E., Schnitzer, J.E., Oh, P., Lisanti, M.P., and Lodish, H.F. (1997). Flotillin and epidermal surface antigen define a new family of caveolae-associated integral membrane proteins. J. Biol. Chem. 272, 13793-13802.

Brasaemle, D.L., Dolios, G., Shapiro, L., and Wang, R. (2004). Proteomic analysis of proteins associated with lipid droplets of basal and lipolytically stimulated 3T3-L1 adipocytes. J. Biol. Chem. 279, 46835-46842.

Breuza, L., Corby, S., Arsanto, J.P., Delgrossi, M.H., Scheiffele, P., and Le Bivic, A. (2002). The scaffolding domain of caveolin 2 is responsible for its Golgi localization in Caco-2 cells. J. Cell Sci. 115, 4457-4467.

Campbell, S.M., Crowe, S.M., and Mak, J. (2001). Lipid rafts and HIV-1: from viral entry to assembly of progeny virions. J. Clin. Virol. 22, 217-227.

Deininger, S.O., Rajendran, L., Lottspeich, F., Przybylski, M., Illges, H., Stuermer, C.A., and Reuter, A. (2003). Identification of teleost Thy- 1 and association with the microdomain/lipid raft reggie proteins in regenerating CNS axons. Mol. Cell. Neurosci. 22, 544-554.

Dermine, J.F., Duclos, S., Garin, J., St-Louis, F., Rea, S., Parton, R.G., and Desjardins, M. (2001). Flotillin-1-enriched lipid raft domains accumulate on maturing phagosomes. J. Biol. Chem. 276, 18507-18512.

Drab, M., Verkade, P., Elger, M., Kasper, M., Lohn, M., Lauterbach, B., Menne, J., Lindschau, C., Mende, F., Luft, F.C.,et al. (2001). Loss of caveolae, vascular dysfunction, and pulmonary defects in caveolin-1 gene-disrupted mice. Science 293, 2449-2452.

Edidin, M. (2003). The state of lipid rafts: from model membranes to cells. Annu. Rev. Biophys. Biomol. Struct. 32, 257-283.

Ehehalt, R., Keller, P., Haass, C., Thiele, C., and Simons, K. (2003). Amyloidogenic processing of the Alzheimer $\beta$-amyloid precursor protein depends on lipid rafts. J. Cell Biol. 160, 113-123.

Fra, A.M., Williamson, E., Simons, K., and Parton, R.G. (1995). De novo formation of caveolae in lymphocytes by expression of VIP21-caveolin. Proc. Natl. Acad. Sci. USA 92, 86558659.

Fujimoto, T., Kogo, H., Ishiguro, K., Tauchi, K., and Nomura, R. (2001). Caveolin-2 is targeted to lipid droplets, a new 'membrane domain' in the cell. J. Cell Biol. 152, 1079-1085.

Galbiati, F., Engelman, J.A., Volonte, D., Zhang, X.L., Minetti, C., Li, M., Hou, H. Jr., Kneitz, B., Edelmann, W., and Lisanti, M.P. (2001). Caveolin-3 null mice show a loss of caveolae, changes in the microdomain distribution of the dystrophin-glycoprotein complex, and t-tubule abnormalities. J. Biol. Chem. 276, 21425-21433.

Glebov, O.O., Bright, N.A., and Nichols, B.J. (2006). Flotillin-1 defines a clathrin-independent endocytic pathway in mammalian cells. Nat. Cell Biol. 8, 46-54.
Hooper, N.M. (2005). Roles of proteolysis and lipid rafts in the processing of the amyloid precursor protein and prion protein. Biochem. Soc. Trans. 33, 335-338.

Kurzchalia, T. (2003). Anthrax toxin rafts into cells. J. Cell Biol. 160, 295-296.

Kurzchalia, T.V., and Parton, R.G. (1999). Membrane microdomains and caveolae. Curr. Opin. Cell Biol. 11, 424-431.

Lang, D.M., Lommel, S., Jung, M., Ankerhold, R., Petrausch, B., Laessing, U., Wiechers, M.F., Plattner, H., and Stuermer, C.A. (1998). Identification of reggie-1 and reggie-2 as plasma membrane-associated proteins which cocluster with activated GPI-anchored cell adhesion molecules in non-caveolar micropatches in neurons. J. Neurobiol. 37, 502-523.

Le Lay, S., Hajduch, E., Lindsay, M.R., Le Liepvre, X., Thiele, C., Ferre, P., Parton, R.G., Kurzchalia, T., Simons, K., and Dugail, I. (2006). Cholesterol-induced caveolin targeting to lipid droplets in adipocytes: a role for caveolar endocytosis. Traffic 7 , 549-561.

Lencer, W.I. (2001). Microbes and microbial toxins: paradigms for microbial-mucosal toxins. V. Cholera: invasion of the intestinal epithelial barrier by a stably folded protein toxin. Am. J. Physiol. Gastrointest. Liver Physiol. 280, G781-786.

Liu, J., Deyoung, S.M., Zhang, M., Dold, L.H., and Saltiel, A.R. (2005). The stomatin/prohibitin/flotillin/HflK/C domain of flotillin-1 contains distinct sequences that direct plasma membrane localization and protein interactions in 3T3-L1 adipocytes. J. Biol. Chem. 280, 16125-16134.

Liu, P., Ying, Y., Zhao, Y., Mundy, D.I., Zhu, M., and Anderson, R.G. (2004). Chinese hamster ovary K2 cell lipid droplets appear to be metabolic organelles involved in membrane traffic. J. Biol. Chem. 279, 3787-3792.

Malaga-Trillo, E., Laessing, U., Lang, D.M., Meyer, A., and Stuermer, C.A. (2002). Evolution of duplicated reggie genes in zebrafish and goldfish. J. Mol. Evol. 54, 235-245.

Martin, S. and Parton, R.G. (2005). Caveolin, cholesterol, and lipid bodies. Semin. Cell Dev. Biol. 16, 163-174.

Neumann-Giesen, C., Falkenbach, B., Beicht, P., Claasen, S., Luers, G., Stuermer, C.A., Herzog, V., and Tikkanen, R. (2004). Membrane and raft association of reggie-1/flotillin-2: role of myristoylation, palmitoylation and oligomerization and induction of filopodia by overexpression. Biochem. J. 378, 509-518.

Ostermeyer, A.G., Paci, J.M., Zeng, Y., Lublin, D.M., Munro, S., and Brown, D.A. (2001). Accumulation of caveolin in the endoplasmic reticulum redirects the protein to lipid storage droplets. J. Cell Biol. 152, 1071-1078.

Parton, R.G. (1996). Caveolae and caveolins. Curr. Opin. Cell Biol. 8, 542-548.

Parton, R.G. (2003). Caveolae - from ultrastructure to molecular mechanisms. Nat. Rev. Mol. Cell Biol. 4, 162-167.

Pol, A., Luetterforst, R., Lindsay, M., Heino, S., Ikonen, E., and Parton, R.G. (2001). A caveolin dominant negative mutant associates with lipid bodies and induces intracellular cholesterol imbalance. J. Cell Biol. 152, 1057-1070.

Pol, A., Martin, S., Fernandez, M.A., Ferguson, C., Carozzi, A., Luetterforst, R., Enrich, C., and Parton, R.G. (2004). Dynamic and regulated association of caveolin with lipid bodies: modulation of lipid body motility and function by a dominant negative mutant. Mol. Biol. Cell 15, 99-110.

Pol, A., Martin, S., Fernandez, M.A., Ingelmo-Torres, M., Ferguson, C., Enrich, C., and Parton, R.G. (2005). Cholesterol and fatty acids regulate dynamic caveolin trafficking through the Golgi complex and between the cell Surface and lipid bodies. Mol. Biol. Cell 16, 2091-2105.

Rajendran, L. and Simons, K. (2005). Lipid rafts and membrane dynamics. J. Cell Sci. 118, 1099-1102.

Rajendran, L., Masilamani, M., Solomon, S., Tikkanen, R., Stuermer, C.A., Plattner, H., and Illges, H. (2003). Asymmetric localization of flotillins/reggies in preassembled platforms confers inherent polarity to hematopoietic cells. Proc. Natl. Acad. Sci. USA 100, 8241-8246. 
Rajendran, L., Honsho, M., Zahn, T.R., Keller, P., Geiger, K.D., Verkade, P., and Simons, K. (2006). Alzheimer's disease $\beta$ amyloid peptides are released in association with exosomes. Proc. Natl. Acad. Sci. USA 103, 11172-11177.

Razani, B., Engelman, J.A., Wang, X.B., Schubert, W., Zhang, X.L., Marks, C.B., Macaluso, F., Russell, R.G., Li, M., Pestell, R.G., et al. (2001). Caveolin-1 null mice are viable but show evidence of hyperproliferative and vascular abnormalities. J. Biol. Chem. 276, 38121-38138.

Schroeder, W.T., Stewart-Galetka, S., Mandavilli, S., Parry, D.A., Goldsmith, L., and Duvic, M. (1994). Cloning and characterization of a novel epidermal cell surface antigen (ESA). J. Biol. Chem. 269, 19983-19991.

Schuck, S., Honsho, M., Ekroos, K., Shevchenko, A., and Simons, K. (2003). Resistance of cell membranes to different detergents. Proc. Natl. Acad. Sci. USA 100, 5795-5800.

Schulte, T., Paschke, K.A., Laessing, U., Lottspeich, F., and Stuermer, C.A. (1997). Reggie-1 and reggie-2, two cell surface proteins expressed by retinal ganglion cells during axon regeneration. Development 124, 577-587.

Shyng, S.L., Heuser, J.E., and Harris, D.A. (1994). A glycolipidanchored prion protein is endocytosed via clathrin-coated pits. J. Cell Biol. 125, 1239-1250.

Simons, K., and Toomre, D. (2000). Lipid rafts and signal transduction. Nat. Rev. Mol. Cell Biol. 1, 31-39.
Simons, K., and Vaz, W.L. (2004). Model systems, lipid rafts, and cell membranes. Annu. Rev. Biophys. Biomol. Struct. 33, 269-295.

Solomon, S., Masilamani, M., Rajendran, L., Bastmeyer, M., Stuermer, C.A., and Illges, H. (2002). The lipid raft microdomain-associated protein reggie-1/flotillin-2 is expressed in human $B$ cells and localized at the plasma membrane and centrosome in PBMCs. Immunobiology 205, 108-119.

Souto, R.P., Vallega, G., Wharton, J., Vinten, J., Tranum-Jensen, J., and Pilch, P.F. (2003). Immunopurification and characterization of rat adipocyte caveolae suggest their dissociation from insulin signaling. J. Biol. Chem. 278, 18321-18329.

Umlauf, E., Csaszar, E., Moertelmaier, M., Schuetz, G.J., Parton, R.G., and Prohaska, R. (2004). Association of stomatin with lipid bodies. J. Biol. Chem. 279, 23699-23709.

Volonte, D., Galbiati, F., Li, S., Nishiyama, K., Okamoto, T., and Lisanti, M.P. (1999). Flotillins/cavatellins are differentially expressed in cells and tissues and form a hetero-oligomeric complex with caveolins in vivo. Characterization and epitopemapping of a novel flotillin-1 monoclonal antibody probe. J. Biol. Chem. 274, 12702-12709.

Received September 21, 2006; accepted November 30, 2006 